\title{
An Algebra of Boolean Processes *
}

\author{
Costas Courcoubetis \\ Department of Computer Science \\ University of Crete \\ Heraklion, Greece
}

\author{
Susanne Graf Joseph Sifakis \\ IMAG-LGI \\ BP 53X \\ F-38041 Grenoble
}

\begin{abstract}
This work has been motivated by the study of the $S / R$ models which allow to represent systems as a set of communicating state machines cooperating through a shared memory.

We show that $S / R$ models can be expressed in terms of a process algebra called Boolean SCCS which is a special case of Milner's SCCS, in the sense that the actions are elements of some boolean algebra. We define for Boolean SCCS an operational and a symbolic semantics modulo strong bisimulation equivalence. A complete axiomatisation of bisimulation and simulation equivalences on this algebra is proposed.

Furthermore, we propose a very general renaming operator, and show by means of examples that it allows the definition of abstractions.
\end{abstract}

\section{Introduction}

Most existing algebraic specification languages for concurrent systems such as process algebras, are based on the communicating processes model. They suppose that a system is composed of a set of components with disjoint state spaces, interacting by exchanging messages. Although of equal importance, models relying upon shared memory communication mechanisms did not attract so much the attention of researchers. A reason might be that the communicating processes model is sufficiently general to represent them. On the other hand, the use of shared memory formalisms leads to compact specifications due to the use of powerful communication mechanisms. By allowing processes to be labelled with complex boolean formulas instead of simple actions, we obtain processes with fewer states since a transition label can represent a set of atomic actions.

Besides obtaining compact specifications, there are other issues which make such formalisms very interesting. They have to do with the possibility of doing reductions at the symbolic level, and in general, the possibility to perform a large part of the verification process at the same level. In order to achieve that, one can use the symbolic manipulation mechanisms provided by the boolean calculus. It is important to note that any reduction at the symbolic level will greatly enhance the applicability of the verification procedures by diminuishing the state explosion effects. This paper attempts to define the notions of symbolic bisimulation and abstraction for such shared memory communicating processes. It also shows that the process algebra paradigm can be directly applied to shared memory models. Interestingly enough, abstraction and renaming in the above models are richer concepts than abstraction and renaming in the traditional communicating processes models.

In order to motivate the reader for using such shared memory formalisms, we start in Section 2 by describing the example of such a formalism which has been successfully used for specifying and verifying large concurrent systems. This is the Selection/Resolution model by R. Kurshan. In Section 3, we give the general definition of boolean transition systems, that is, transition systems whose labels are elements of a boolean algebra, and which is our model of the shared memory communicating processes.

"This work has been partially supported by ESPRIT Basic Research Action 'Spec' 
Section 4 presents an algebra of boolean processes, for which two different semantics in terms of boolean transition systems modulo bisimulation are defined: an 'operational' one, whose models are usual action-labelled transition systems, and a 'symbolic' one, whose models are transition systems whose labels are boolean expressions. We give notions of strong bisimulation for both semantics and show that they coincide on terms. Furthermore, we propose a complete axiomatisation of bisimulation on terms, showing that our algebra is a particular case of $S C C S$ with boolean actions. In this section, we give also some results on renaming functions and illustrate their use for the definition of abstractions by an example. In Section 5 , we define notions of simulation preorder and equivalence.

\section{The Selection/Resolution Model}

\subsection{Informal presentation}

The selection/resolution $(S / R)$ model [AKS83a,AC85,GK80,Ku90,ABM86a] provides a method of describing a system as a set of coordinating finite state machines. Experience has shown that complex systems can be specified by using this model, and there are currently tools which automatically verify properties of the behaviours of such formal specifications, managing systems with millions of reachable states [Ku90]. An important feature is the fact that the coupling between machines is described in terms of predicates. This helps in many cases to obtain concise and understandable specifications.

A system is decomposed into a set of simple components; each component or process is an edge labelled directed graph (see Figure 1). The vertices of this graph are states of the process; each directed edge describes a transition corresponding to one computation step. In each state, a process can nondeterministically choose from a set of selections, which are essentially values of a shared memory used for synchronization. In fact, there is a shared memory in the system consisting of a finite number of variables ranging over a finite domain. With each process is associated a subset of selection variables which are distinct for each process. A process can read all variables, whereas it can update only its own selection variables (selections are enclosed in braces next to the states in Figure 1, an example in which the selection functions are all deterministic).

A computation step of the system consists of a selection followed by a resolution phase. The selection of a process consists in choosing a value for each one of its selection variables. The resolution is done by calculating the global selection, i.e., the vector of all the current selections of the processes. Each process checks which transitions are consistent with the current selections of all processes, and then chooses one of these enabled transitions.

\subsection{The $S / R$-processes}

Notation 2.1 $\mathcal{B}$ is a boolean algebra with $\vee, \wedge,-, \Rightarrow$ denoting respectively disjunction, conjunction, complementation and implication. By convention, 0 and 1 represent respectively the bottom and the top element of $\mathcal{B}$ and atoms $(\mathcal{B})$ is the set of atoms of $\mathcal{B}$.

Definition 2.2 ( $S / R$-process)

An $S / R$-process on a boolean algebra $\mathcal{B}$ is a triplet $S R=(Q, \delta, \sigma)$, where

- $Q$ is a set of states,

- $\delta: Q \times Q \mapsto \mathcal{B}$ is a transition function,

- $\sigma: Q \mapsto \mathcal{B}$ is a selector function.

An $S / R$-process can be represented by a state- and edge-labelled directed graph whose vertices are the states. There is an edge from state $q$ to $q^{\prime}$ labelled by $\ell$ iff $\delta\left(q, q^{\prime}\right)=\ell$ and $\ell \neq 0$.

Definition 2.3 (parallel composition on $S / R$-processes)

Let $S R_{i}=\left(Q_{i}, \delta_{i}, \sigma_{i}\right)$ for $i=1,2$ be two $S / R$-processes on $\mathcal{B}$. The parallel composition of $S R_{1}$ and $S R_{2}$ is the $S / R$-process $S R_{1} \times S R_{2}=(Q, \delta, \sigma)$ where, 
- $Q=Q_{1} \times Q_{2}$,

- $\sigma\left(q_{1}, q_{2}\right)=\sigma_{1}\left(q_{1}\right) \wedge \sigma_{2}\left(q_{2}\right)$

- $\delta\left(\left(q_{1}, q_{2}\right),\left(q_{1}^{\prime}, q_{2}^{\prime}\right)\right)=\delta_{1}\left(q_{1}, q_{1}^{\prime}\right) \wedge \delta_{2}\left(q_{2}, q_{2}^{\prime}\right)$

Example We demonstrate the use of the $S / R$ model for the description of a simple modulo 8 counter whose output (the integers between 0 and 7 ) is represented by 3 boolean variables $y_{0}, y_{1}, y_{2}$. Its input, the signal incrementing the counter, is represented by a boolean variable $x$.

The counter is modelled as the parallel composition of three $S / R$-processes $S R_{0}, S R_{1}, S R_{2}$ with selection variables respectively $y_{0}, y_{1}$ and $y_{2}$ (Figure 1 ).

Such specifications can be treated by tools such as COSPAN and SPANNER and prove properties of the infinite sequences of the global memory assignments (see [ABM86a,b], [ACW90], [KK86]).

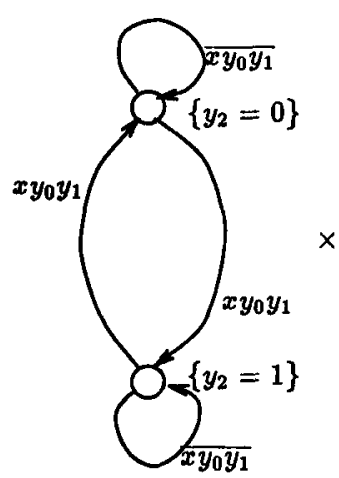

$S R_{2}$

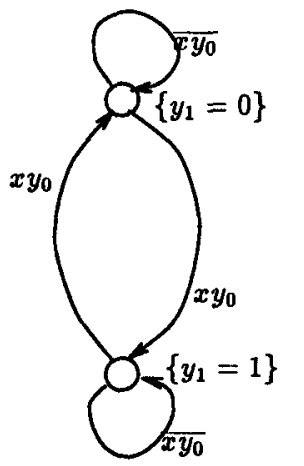

$S R_{1}$

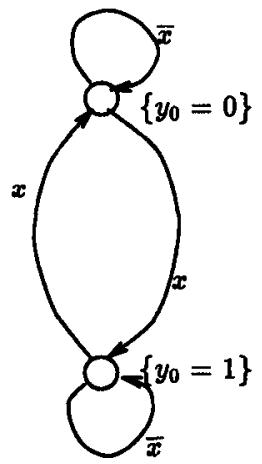

$S R_{0}$

Figure 1: A modulo 8 counter

\section{Boolean Transition Systems}

In this section, we define boolean transition systems, which are transition systems labelled by elements of a boolean algebra $\mathcal{B}$. They differ from $S / R$-processes only by the fact that they have no labels on states. We show the relationship between these two models.

\section{1 $S / R$-processes as boolean transition systems (BTS)}

Definition 3.1 (Boolean transition systems)

$A$ boolean transition system on a boolean algebra $\mathcal{B}$ is a pair $S=(Q, \rightarrow)$, where

- $Q$ is a set of states,

- $\rightarrow \subseteq Q \times(\mathcal{B}-\{0\}) \times Q$ is the transition relation, where we write $q^{\ell} \stackrel{\longrightarrow}{\rightarrow} q^{\prime}$ for $\left(q, \ell, q^{\prime}\right) \in \rightarrow$.

Definition 3.2 With an $S / R$-process $S R=(Q, \delta, \sigma)$ on $\mathcal{B}$, we associate a $\mathcal{B} T S, \mathcal{B t s}(S R)=(Q, \rightarrow)$, where $\rightarrow$ is defined as the least relation, subset of $Q \times(B-\{0\}) \times Q$, such that:

$$
\left(\delta\left(q, q^{\prime}\right)=\ell \text { and } \ell \wedge \sigma(q) \neq 0\right) \text { implies } q^{\ell \wedge \sigma(q)} q^{\prime} .
$$

The parallel composition on $S / R$-processes can easily be translated into the parallel composition of boolean transition systems: 
Definition 3.3 (parallel composition on $\mathcal{B} T S$ )

Let $S_{i}=\left(Q_{i}, \rightarrow_{i}\right)$ for $i=1,2$ be two BTSs. The parallel composition of $S_{1}$ and $S_{2}$ is the BTS, $S=S_{1} \times S_{2}=(Q, \rightarrow)$ where,

- $Q=Q_{1} \times Q_{2}$,

- $\left(q_{1}, q_{2}\right) \stackrel{\ell}{\longrightarrow}\left(q_{1}^{\prime}, q_{2}^{\prime}\right)$ iff $\left(\ell \neq 0\right.$ and $\exists \ell_{1}, \ell_{2} .\left(\ell=\ell_{1} \wedge \ell_{2}\right.$ and $q_{i} \stackrel{l_{i}}{\longrightarrow} q_{i}^{\prime}$ for $\left.\left.i=1,2\right)\right)$.

In fact, we obtain in a straight forward manner the following proposition:

Proposition 3.4 For any $S / R$-processes $S R_{1}, S R_{2}$,

$\mathcal{B} t s\left(S R_{1} \times S R_{2}\right)=\mathcal{B} t s\left(S R_{1}\right) \times \mathcal{B} t s\left(S R_{2}\right)$.

Example: translation of an $S / R$-process into a $\mathcal{B} T S$

Consider again the counter of Figure 1 given as an $S / R$ model. The corresponding $B T S$, $B t s\left(S R_{0} \times S R_{1} \times S R_{2}\right)$ is obtained by calculating the parallel composition $\mathcal{B} t s\left(S R_{0}\right) \times \mathcal{B} t s\left(S R_{1}\right) \times \mathcal{B} t s\left(S R_{2}\right)$ as shown in Figure 2.
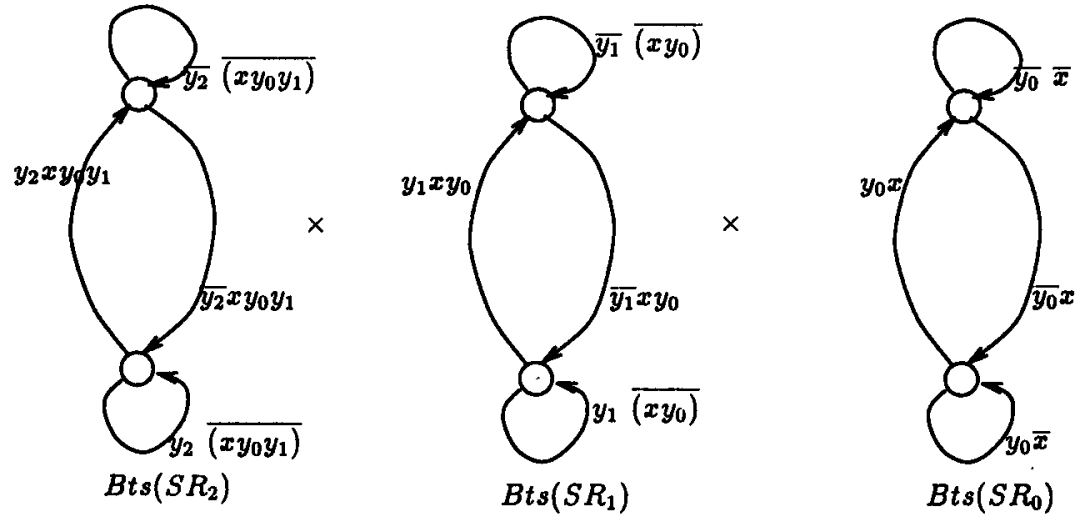

Figure 2: The modulo 8 counter as BTS

We introduce hereafter some useful notations for boolean transition systems.

Definition 3.5 Let $S=(Q, \rightarrow)$ be a B TS and $q \in Q$. Then,

1. The enabling condition of a state $q$, enable $(q)$ is the boolean expression $\operatorname{enable}(q)=V_{\exists q^{\prime} \cdot q} \stackrel{\ell_{i}}{\longrightarrow} q_{q^{\prime}} \ell_{i}$.

2. $q$ is called finitely branching iff $\forall \ell \in \mathcal{B}$ there is a finite number of labels $\ell^{\prime}$ such that $\left(q \stackrel{\ell^{\prime}}{\longrightarrow}\right.$ and $\left.\ell \Rightarrow \ell^{\prime}\right)$.

3. $q$ is called deterministic iff $\forall \ell_{1}, \ell_{2} q \stackrel{\ell_{1}}{\rightarrow} q_{1}$ and $q \stackrel{\ell_{2}}{\rightarrow} q_{2}$ implies $\left(\ell_{1}=\ell_{2}\right.$ and $\left.q_{1}=q_{2}\right)$ or $\ell_{1} \wedge \ell_{2}=0$.

4. $q$ is called complete iff enable $(q)=1$

5. $q$ is called canonical iff $\forall q^{\prime}\left(q \stackrel{\ell}{\longrightarrow} q^{\prime}\right.$ implies $\left.\ell \in \operatorname{atoms}(\mathcal{B})\right)$.

$S$ is respectively called finitely branching, deterministic, complete or canonical if all its states have the corresponding property. 


\section{Boolean SCCS, an algebra for boolean transition systems}

In this section, we define a process algebra that can be considered as a particular case of SCCS [Mi83], i.e., a process algebra with a synchronous parallel operator. Its action operators are the elements of some boolean algebra $\mathcal{B}$. Processes of this algebra have boolean transition systems as underlying models. We study in particular bisimulation semantics for this algebra.

Notation 4.1 (renaming function)

Let $\mathcal{B}$ be a boolean algebra. Any mapping $\phi: \mathcal{B} \mapsto \mathcal{B}$ satisfying $\phi(0)=0$ and which is distributive over disjunction $\left(\forall \ell_{1}, \ell_{2} \in \mathcal{B} . \phi\left(\ell_{1} \vee \ell_{2}\right)=\phi\left(\ell_{1}\right) \vee \phi\left(\ell_{2}\right)\right)$ is called a renaming function on $\mathcal{B}$.

Definition 4.2 (Syntax of $B$ SCCS)

Let $\mathcal{B}$ be a boolean algebra, $\phi$ a renaming function and $Z$ a set of variables. Represent by $\ell$ and $z$ respectively, elements of $\mathcal{B}$ and $Z$. Consider the term language defined by the following grammar:

$$
\begin{aligned}
& t_{s}::=\oslash|z| \ell t_{s}\left|t_{s}+t_{s}\right| \text { recz.t. } \\
& \left.t::=t_{s}|t \times t| t \mid \phi\right]|\ell t| t+t
\end{aligned}
$$

We call BSCCS the sub-algebra of the closed terms, named also processes. As usually, a term is called guarded if in any subterm of the form recz.t all occurrences of $z$ in $t$ are in the scope of an action-operator $\ell$.

Notice that a term of $\mathcal{B S C C S}$ has no occurrences of $\times$ within the scope of a recursion operator as we want to restrict ourselves to regular processes.

\subsection{Operational semantics}

Definition 4.3 (operational semantics)

For $\ell \in \mathcal{B}, a, a^{\prime} \in$ atoms $(\mathcal{B}), t_{1}, t_{2}, t \in \mathcal{B S C C S}, \phi$ a renaming function and $z$ a process variable, the transition relation $\sim$ on BSCCS is defined as the smallest relation specified by the following rules.

1. $\ell \stackrel{a}{\rightarrow} t \quad$ iff $\quad a \Rightarrow \ell$

2. $t_{1} \stackrel{a}{\sim} t_{1}^{\prime}$ implies $t_{1}+t_{2} \stackrel{a}{\sim} t_{1}^{\prime}$ and $\stackrel{t_{2}}{\stackrel{a}{\sim}} t_{2}^{\prime} \quad$ implies $t_{1}+t_{2} \stackrel{a}{\sim} t_{2}^{\prime}$

3. $t_{1} \stackrel{a}{\sim} t_{1}^{\prime} \wedge t_{2} \stackrel{a}{\sim} t_{2}^{\prime}$ implies $t_{1} \times t_{2} \stackrel{a}{\sim} t_{1}^{\prime} \times t_{2}^{\prime}$

4. $t \stackrel{a}{\sim} t^{\prime} \wedge \quad a^{\prime} \Rightarrow \phi(a) \quad$ implies $t[\phi] \stackrel{a^{\prime}}{\sim} t^{\prime}[\phi]$

5. $t \stackrel{a}{\rightarrow} t^{\prime}$ implies recz.t $\stackrel{a}{\sim} t^{\prime}[$ recz.t $/ z]$

These rules associate with any term of Boolean SCCS a canonical BTS by defining for any operator an operator on BTSs. The set of atoms can also be considered as the set of labels of a usual labelled transition system.

\section{Remarks:}

- If the boolean algebra $\mathcal{B}$ is generated by a set of boolean variables, then atoms can also be considered as valuations, i.e. functions associating boolean values with the boolean variables generating the algebra,

- The renaming operator $[\phi]$ plays the role of both an abstraction and a restriction operator, depending on the nature of $\phi$. If $\phi$ associates 0 with some atoms, and leaves the others unchanged, then it corresponds to a restriction operator. The use of renaming as an abstraction operator will be illustrated later (see Section 4.5).

We are interested in strong bisimulation semantics on $\mathcal{B}$ TS. 
Notation 4.4 (strong bisimulation $\sim$ )

- We denote by $\sim$ the strong bisimulation relation induced by the transition relation $\leadsto$.

- We denote as usual by $\sim_{i}$ the bisimulation up to depth $i$. We have $\sim=\bigcap_{i=1}^{\infty} \sim_{i}$ as even for infinite $\mathcal{B}$ any term has only a finite number of 'a-derivations' for any a $\in$ atoms $(\mathcal{B})$.

As in [Mi83], we obtain the following proposition. For the renaming operator to preserve bisimulation it is necessary that the renaming functions are strict and distributive over disjunction.

Proposition $4.5 \sim$ is a congruence on BSCCS.

\subsection{Symbolic semantics for Boolean SCCS}

In this section, we give a different operational semantics associating an arbitrary $\mathcal{B} T S$ with a term of Boolean SCCS. We define a symbolic bisimulation which is proven to coincide with strong bisimulation on $\mathcal{B S C C S}$.

Definition 4.6 (symbolic semantics)

For $\ell_{1}, \ell_{2}, \ell \in \mathcal{B}, t_{1}, t_{2}, t \in \mathcal{B S C C S}$ and $z$ a process variable, let $\rightarrow$ be the transition relation, defined as the smallest relation specified by the following rules.

1. $\ell t \stackrel{\ell}{\longrightarrow}$ iff $\ell \neq 0$

2. $t_{1} \stackrel{\iota}{\longrightarrow} t_{1}^{\prime}$ implies $t_{1}+t_{2} \stackrel{\ell}{\longrightarrow} t_{1}^{\prime} \quad$ and $\quad t_{2} \stackrel{\ell}{\longrightarrow} t_{2}^{\prime} \quad$ implies $\quad t_{1}+t_{2} \stackrel{\ell}{\longrightarrow} t_{2}^{\prime}$

3. $t_{1} \stackrel{\ell_{1}}{\longrightarrow} t_{1}^{\prime} \wedge t_{2} \stackrel{\ell_{2}}{\longrightarrow} t_{2}^{\prime} \wedge\left(\ell_{1} \wedge \ell_{2} \neq 0\right) \quad$ implies $t_{1} \times t_{2} \stackrel{\ell_{1} \wedge \ell_{2}}{\longrightarrow} t_{1}^{\prime} \times t_{2}^{\prime}$

4. $t \stackrel{\ell}{\longrightarrow} t^{\prime} \wedge \phi(\ell) \neq 0 \quad$ implies $\quad t[\phi] \stackrel{\phi(\ell)}{\longrightarrow} t^{\prime}[\phi]$

5. $t \stackrel{\ell}{\longrightarrow} t^{\prime}$ implies recz.t $\stackrel{\ell}{\longrightarrow} t^{\prime}[$ recz.t/z]

\section{Remarks:}

- As for the operational semantics, these rules allow to associate in an obvious manner with any term of Boolean SCCS a BTS (not necessarily a canonical one) by defining for any operator an operator on $\mathcal{B}$ TSs.

- Conversely, with any finite $\mathcal{B}$ TS can be associated a process in an obvious manner. Thus, in the sequel we identify a term of Boolean SCCS with its corresponding boolean transition system.

- Therefore, the notations of Definition 3.5 can be applied to terms. We say for a term $t$, enable $(t)=\ell, t$ is respectively finitely branching, deterministic, complete or canonical if and only if this is the case for the $\mathcal{B T S}$ associated via its symbolic semantics.

Definition 4.7 (symbolic bisimulation)

Let be $t_{1}, t_{2} \in \mathcal{B S C C S}$. Then, $\simeq$ is defined as the largest symmetric relation, solution of $\Phi(\mathcal{R})=\mathcal{R}$, where

$\left(t_{1}, t_{2}\right) \in \Phi(\mathcal{R})$ iff

$\forall \ell \in \mathcal{B} \forall t_{1} \in \mathcal{B S C C S}\left(t_{1} \stackrel{\ell}{\longrightarrow} t_{1}^{\prime}\right.$ implies $\exists I .\left(\left(\ell \Rightarrow V_{i \in I} \ell_{i}\right)\right.$ and $\forall i \in I \exists t_{2 i} \cdot\left(t_{2} \stackrel{\ell_{i}}{\longrightarrow} t_{2 i}\right.$ and $\left.\left.\left.\left(t_{1}^{\prime}, t_{2 i}\right) \in \mathcal{R}\right)\right)\right)$

As usually, we write $t_{1} \simeq t_{2}$ instead of $\left(t_{1}, t_{2}\right) \in \simeq$ and we say that $t_{1}$ symbolically bisimulates $t_{2}$. 


\section{Remarks:}

- $t_{1} \simeq t_{2}$ implies enable $\left(t_{1}\right)=$ enable $\left(t_{2}\right)$.

- Any complete term symbolically bisimulates the process $\mathbb{I}$, defined as $\mathbb{I}=$ recz.1z.

The characterization of bisimulation as the intersection of bisimulations up to depth $i$ can also be shown for symbolic bisimulation.

Proposition $4.8 \simeq=\bigcap_{i=1}^{\infty} \simeq_{i}$, where

- $\simeq_{0}=\mathcal{B S C C S} \times \mathcal{B S C C S}$

- $\simeq_{i+1}=\Phi\left(\simeq_{i}\right) \forall i \in \mathbb{N}, i>0$

This result can be used to compute $\simeq$, and thus to reduce processes, and also finite $\mathcal{B} T S$ s. In Figure 3, we give two symbolically bisimilar BTSs. The small one is the quotient modulo $\simeq$ of the other.

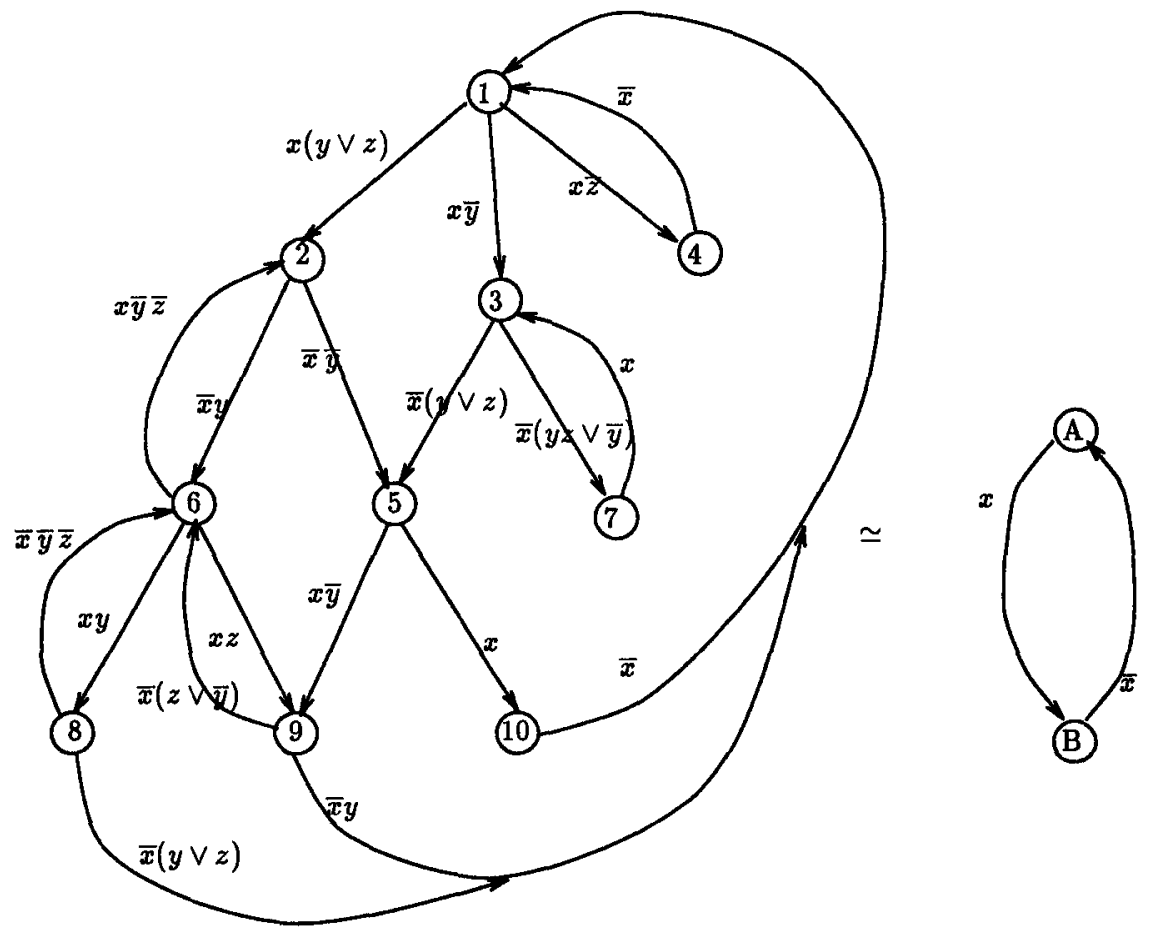

Figure 3: Reduction modulo symbolic bisimulation

Proposition 4.9 Symbolic and strong bisimulation agree on Boolean SCCS, i.e. $\simeq=\sim$.

Proof: We prove that for any $i, \sim_{i}=\simeq_{i}$ by induction. The proof is easy if we use the observation that $\forall a \in \operatorname{atoms}(\mathcal{B}) t \stackrel{a}{\sim} t^{\prime}$ iff $\exists \ell \in \mathcal{B} .\left(a \Rightarrow \ell\right.$ and $\left.t \stackrel{\ell}{\longrightarrow} t^{\prime}\right)$

Proposition $4.10 \simeq$ is a congruence on $\mathcal{B S C C S}$.

Proof: Direct from the proposition above and the fact that $\sim$ is a congruence on BSCCS. 


\subsection{Results for deterministic processes}

For deterministic processes the definition of $\simeq$ can be simplified in the following manner:

Definition 4.11 Let $\simeq^{d}$ be the largest symmetric relation on $\mathcal{B S C C S}$, solution of $\Phi_{1}(\mathcal{R})=\mathcal{R}$, where $\left(t_{1}, t_{2}\right) \in \Phi_{1}(\mathcal{R})$ iff

- enable $\left(t_{1}\right)=$ enable $\left(t_{2}\right)$

- $t_{1} \stackrel{\iota}{\longrightarrow} t_{1}^{\prime}$ implies $\forall \ell^{\prime}, t_{2}^{\prime}\left(\left(\ell \wedge \ell^{\prime} \neq 0\right.\right.$ and $\left.t_{2} \stackrel{\ell^{\prime}}{\rightarrow} t_{2}^{\prime}\right)$ implies $\left.\left(t_{1}^{\prime}, t_{2}^{\prime}\right) \in \mathcal{R}\right)$

Proposition 4.12 (characterization of $\simeq$ on deterministic processes)

For $t_{1}, t_{2} \in \mathcal{B S C C S}, t_{1}, t_{2}$ nondeterministic $\left(i_{1} \simeq t_{2}\right.$ iff $\left.t_{1} \simeq{ }^{d} t_{2}\right)$.

Proof: We have already noticed that $t_{1} \simeq t_{2}$ implies enable $\left(t_{1}\right)=$ enable $\left(t_{2}\right)$. Furthermore, the definition of $\simeq$ says that for any $\ell$-transition of $t_{1}$ leading to $t_{1}^{\prime}$, there exists a set of transitions from $t_{2}$ whose labels cover $\ell$ and which lead to equivalent terms. For a deterministic process the set of transitions whose labels cover $\ell$ is unique.

In this case the condition in the definition of $\simeq, \forall \ell \in \mathcal{B} \exists I,\left\{t_{i}\right\} \cdot\left(\left(\vee_{i \in I} \ell_{i} \Rightarrow \ell\right)\right.$ and $\left.\forall i \in I t \stackrel{\ell_{i}}{\longrightarrow} t_{i}\right)$ is equivalent to $\forall \ell \in \mathcal{B}\left(\exists \ell^{\prime} \in \mathcal{B}, t^{\prime} \in \mathcal{B S C C S}\right.$. $\left(\ell \wedge \ell^{\prime} \neq 0\right.$ and $\left.\left.t \stackrel{\ell^{\prime}}{\longrightarrow} t^{\prime}\right)\right)$.

The fact that $\simeq$ and $\simeq^{d}$ coincide, is easy to deduce from this observation.

Notice that for the comparison of two terms $t$ and $t^{\prime}$, it is sufficient that one of them is deterministic in order that $\simeq$ and $\simeq^{d}$ coincide. Furthermore, the relation $\simeq^{d}$ gives rise to a simpler verification algorithm.

\subsection{An axiomatisation of symbolic bisimulation on Boolean SCCS}

The axioms and rules characterizing $\simeq$ on Boolean SCCS consist of the axioms characterizing strong bisimulation on SCCS and some additional axioms due to the laws of the action set $\mathcal{B}$.

Theorem 4.13 (axiomatization)

The axiomatization given in Table 1 is sound and complete for $\simeq$ on Boolean SCCS.

Proof: The proof of soundness is standard, except for the axioms concerning renaming, for which we need the fact that $\phi$ is strict and distributes over disjunction.

The completeness can be deduced from the completeness of the axioms (1), (2) and (11) to (13) for strong bisimulation on terms in canonical form obtained in the following manner.

In a first step, a term is transformed into an equivalent one without occurrences of $x$ and renaming operators by means of the axioms (4) to (10), commutativity and associativity. In a second step, such a term can be transformed by using (14) to (16) into canonical form, in which the only action names are atoms of $\mathcal{B}$.

We have already shown that $\simeq$ coincides with strong bisimulation, and on canonical terms strong bisimulation can be characterized by the axioms and rules (1),(2) and (11) to (13) [Mi84].

\subsection{Some results on renaming}

In the following propositions we give some sufficient conditions on functions $\phi$ in order that the corresponding renaming operators $[\phi]$ preserve particular properties of terms.

Proposition 4.14 Let $\phi$ be a renaming function on $\mathcal{B}$.

$\forall t_{1}, t_{2} \in \mathcal{B S C C S}\left(t_{1} \times t_{2}\right)[\phi]=t_{1}[\phi] \times t_{2}[\phi]$, i.e., $[\phi]$ distributes over $\times$, iff

$\forall \ell_{1}, \ell_{2} \in \mathcal{B} \phi\left(\ell_{1} \wedge \ell_{2}\right)=\phi\left(\ell_{1}\right) \wedge \phi\left(\ell_{2}\right)$, i.e. $\phi$ distributes over conjunction. 


\section{(1) Axioms of SCCS:}

1. + is commutative, associative and idempotent,

2. $t+\emptyset=t$

3. $x$ is commutative and associative

4. $t \times \oslash=\varnothing$

5. $t \times\left(t_{1}+t_{2}\right)=\left(t \times t_{1}\right)+\left(t \times t_{2}\right)$

6. $\ell_{1} t_{1} \times \ell_{2} t_{2}=\left(\ell_{1} \wedge \ell_{2}\right)\left(t_{1} \times t_{2}\right)$

7. $\mathbb{1} \times t=t$

8. $\oslash[\phi]=\varnothing$

9. $(a t)[\phi]=a(t[\phi])$

10. $\left(t_{1}+t_{2}\right)[\phi]=t_{1}[\phi]+t_{2}[\phi]$

11. $\operatorname{recz} .\left(z+t^{\prime}\right)=\operatorname{rec} z \cdot t^{\prime}$

12. $r e c z . t=t[$ recz.t $/ z]$

13. $t^{\prime}=t\left[t^{\prime} / z\right]$ implies $t^{\prime}=$ recz.t provided that $t^{\prime}$ is guarded

(2) Axioms and rules specific to $\mathcal{B S C C S}$ :

14. $0 t=\varnothing$

15. $\ell_{1} t+\ell_{2} t=\left(\ell_{1} \vee \ell_{2}\right) t$

16. $\ell_{1} \equiv \ell_{2}$ implies $\ell_{1} t=\ell_{2} t$

Table 1: Axiomatisation of $\simeq$ on $\mathcal{B S C C S}$

Notice that distributivity over conjunction is a very strong requirement for a renaming function, and the renaming functions used for abstraction of our example given at the end of the section do not have this property.

Proposition 4.15 Let $\phi$ be a renaming function on $\mathcal{B}$ and $t$ a term of Boolean SCCS.

1. If $\phi$ is increasing, i.e. $\forall \ell \in \mathcal{B}(\ell \Rightarrow \phi(\ell))$, then

$[\phi]$ preserves completeness of $t$, i.e. if $t$ is complete then $t[\phi]$ is also complete.

2. If $\phi$ is such that $\left(\forall \ell_{1}, \ell_{2} \in \mathcal{B} \ell_{1} \wedge \ell_{2}=0\right.$ implies $\left.\phi\left(\ell_{1}\right) \wedge \phi\left(\ell_{2}\right)=0\right)$ then

$[\phi]$ preserves determinism of $t$, i.e. if $t$ is deterministic then $t[\phi]$ is also deterministic.

3. If $\phi$ maps atoms to atoms, i.e. $\phi$ : atoms $(\mathcal{B}) \mapsto$ atoms $(\mathcal{B})$, then

$[\phi]$ preserves canonicity of $t$, i.e. if $t$ is canonical then $t[\phi]$ is also canonical.

Notice that the condition of (3) implies the condition of (2). 


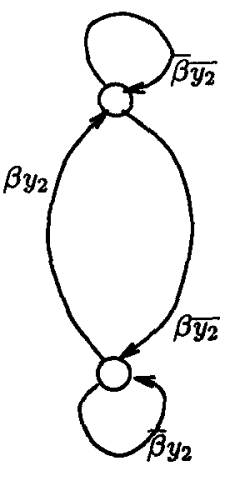

$C\left(\beta, y_{2}, 1\right)$
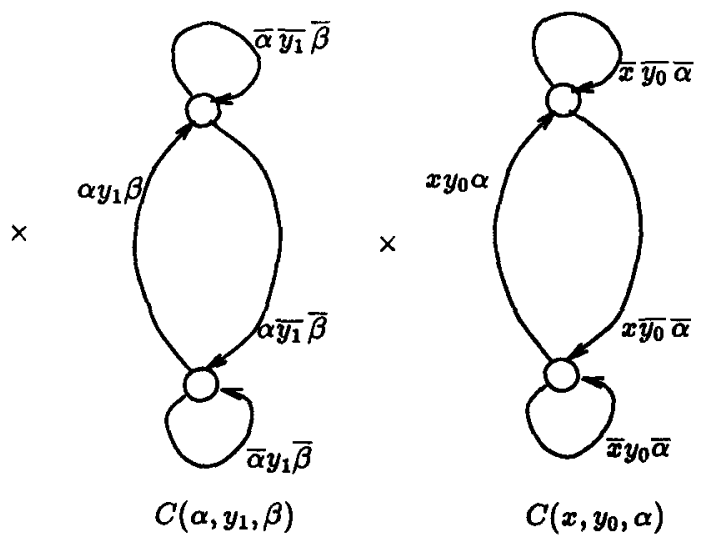

Figure 4: $C_{8}$, a modulo 8 counter

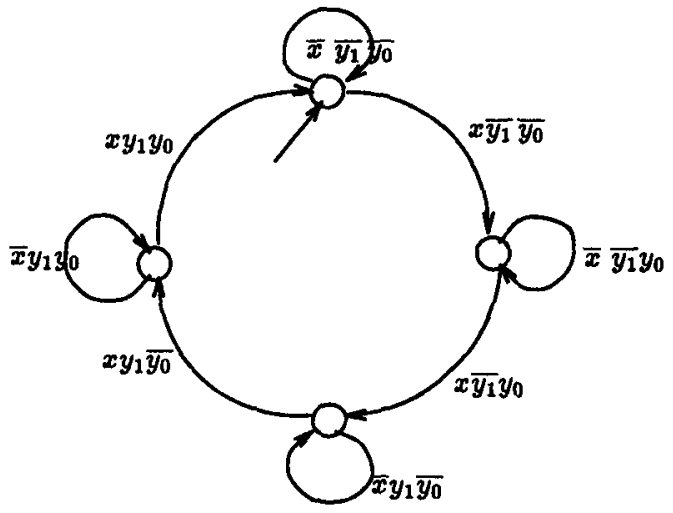

Figure 5: The reduced $\mathcal{B T S}$ of $C_{8}\left[\phi_{1}\right]\left[\phi_{2}\right]$

An Example: modulo 8 counter (see [Ma91])

In this example, we illustrate the use of renaming functions to obtain abstractions. Consider again a modulo 8 counter, defined in a slightly different manner than in Section $3 . C_{8}$ is defined as the parallel composition $C_{B}=C\left(\beta, y_{2}, 1\right) \times C\left(\alpha, y_{1}, \beta\right) \times C\left(x, y_{0}, \alpha\right)$ where the subterms $C\left(v_{1}, v_{2}, v_{3}\right)$, defined in Figure 4, represent modulo 2 counters changing their state on input signal $v_{1}$ with state variable $v_{2}$ and output variable $v_{3}$, representing the overflow bit. The observable variables of the modulo 8 counter are the global input $x$ and the state variables $y_{2}, y_{1}, y_{0}$ whereas $\alpha$ and $\beta$ are only used for synchronization. The renaming function $\phi_{1}$ defined by

$$
\begin{aligned}
& \phi_{1}\left(\ell\left(x, y_{2}, y_{1}, y_{0}, \alpha, \beta\right)\right)=\exists \alpha \beta \ell\left(x, y_{2}, y_{1}, y_{0}, \alpha, \beta\right) \equiv \\
& \quad \ell\left(x, y_{2}, y_{1}, y_{0}, 0,0\right) \vee \ell\left(x, y_{2}, y_{1}, y_{0}, 0,1\right) \vee \ell\left(x, y_{2}, y_{1}, y_{0}, 1,0\right) \vee \ell\left(x, y_{2}, y_{1}, y_{0}, 1,1\right)
\end{aligned}
$$

allows to make abstraction from the overflow variables $\alpha$ and $\beta$.

The $\mathcal{B T S}$ corresponding to $C_{8}\left[\phi_{1}\right]$ has 8 states and cannot be reduced modulo symbolic bisimulation, but its boolean expressions are simpler than that of the $\mathcal{B T S}$ of $C_{8}$. 
Consider the renaming function $\phi_{2}$

$\phi_{2}\left(\ell\left(x, y_{2}, y_{1}, y_{0}\right)\right)=\exists y_{2} \ell\left(x, y_{2}, y_{1}, y_{0}\right) \equiv \ell\left(x, 0, y_{1}, y_{0}\right) \vee \ell\left(x, 1, y_{1}, y_{0}\right)$

which applied to $C_{8}\left[\phi_{1}\right]$ allows to abstract from $y_{2}$.

The boolean transition system corresponding to the process $C_{8}\left[\phi_{1}\right]\left[\phi_{2}\right]$ can be reduced to the one presented in Figure 5 and corresponds clearly to a counter modulo 4.

\section{Simulation preorders and equivalences on $\mathcal{B S C C S}$}

Bisimulation is a strong equivalence, and if we are interested in verifying safety properties much weaker equivalences are interesting [BGFRS90]. In this section, we study simulation preorders and the equivalences they introduce on Boolean SCCS.

Definition 5.1 (simulation preorder $\complement^{s}$ )

$\forall t_{1}, t_{2} \in \mathcal{B S C C S} t_{1} \complement^{\mathbf{s}} t_{2}$

$t_{1} \stackrel{\ell}{\longrightarrow} t_{1}^{\prime}$ implies $\exists I .\left(\left(\ell \Rightarrow V_{i \in I} \ell_{i}\right)\right.$ and $\forall i \in I \exists t_{2 i} \cdot\left(t_{2} \stackrel{\ell_{i}}{\longrightarrow} t_{2 i}\right.$ and $\left.\left.t_{1}^{\prime} \sqsubseteq^{s} t_{2 i}\right)\right)$

The simulation equivalence induced by $\complement^{*}$ is denoted by $\simeq^{*}$.

Remark: As in the case of bisimulation, it can be shown that the above defined simulation preorder coincides on canonical $\mathcal{B} T S$ with the usual simulation preorder.

Proposition 5.2 (characterization of $\sqsubseteq^{\circ}$ )

$\forall t \in \mathcal{B S C C S}\left\{t^{\prime} \in \mathcal{B S C C S} \mid t^{\prime} \underline{\complement}^{\circ} t\right\}=\left\{t^{\prime} \mid \exists t^{\prime \prime} \in \mathcal{B S C C S} . t^{\prime} \simeq{ }^{*} t^{\prime \prime} \times t\right\}$

1. All axioms of Table 1 , where each equation $t_{1}=t_{2}$ stands for two equations $t_{1} \leq t_{2}$ and $t_{2} \leq t_{1}$

2. $\varnothing \leq t$

3. $t_{1} \leq t_{2}\left[t_{1} / z\right]$ implies $t_{1} \leq$ recz. $t_{2}$ provided $z$ guarded in $t_{2}$

4. $t_{2}\left[t_{1} / z\right] \leq t_{1}$ implies recz.t $t_{2} \leq t_{1}$ provided $z$ guarded in $t_{2}$

5. $\ell_{1} \Rightarrow \ell_{2}$ implies $\ell_{1} t \leq \ell_{2} t$

Table 2: Axiomatisation of $\simeq s$ on Boolean SCCS

Proposition 5.3 (Axiomatization of $\coprod^{*}$ )

The axiomatization given in Table 2 is sound and complete for $\underline{\subseteq}^{*}$ on Boolean SCCS.

Proof: The soundness of this axiomatisation is easy to check. The completeness proof is very similar to the one of Theorem 4.13. As before, each term can be transformed into one in canonical form. In [BGFRS90] it has been shown for a term algebra isomorphic to the subalgebra of canonical terms that the above axiomatization (without rule (5) and based on the axioms of SCCS only) characterizes completely the usual simulation preorder. 


\section{Conclusion}

This work establishes a connection between the $S / R$ model and process algebras. For this, we introduce boolean transition systems, an extension of ordinary transition systems.

We believe that the Boolean Process Algebra and its underlying model deserve a further study as such, independently of the $S / R$ model. In fact, they seem to be fairly appropriate formalisms to describe hardware and in general finite systems where data are coded by boolean variables.

Furthermore, symbolic bisimulation allows compare descriptions given by state transition models where labels represent sets of actions. The two given semantics show that boolean processes are more abstract.

It would be interesting to introduce weaker equivalences, such as stuttering equivalence on these models. Another interesting question would be to characterize the renaming functions introducing interesting abstraction criteria.

\section{References}

[ABM86a] S. Aggarwal, D. Barbara, K. Z. Meth. "SPANNER - A Tool for the Specification, Analysis, and Evaluation of Protocols," IEEE Trans. on Software Engineering (to appear).

[AC85] S. Aggarwal, C. Courcoubetis. "Distributed Implementation of a Model of Communication and Computation," Proceedings of the Int. Conf. on System Sciences, January, 1985.

[AKS83a] S. Aggarwal, R. P. Kurshan, K. K. Sabnani. "A Calculus for Protocol Specification and Validation," in Protocol Specification, Testing and Verification III, North-Holland, 1983.

[Ku90] R. Kurshan, "Analysis of Discrete Event Coordination". LNCS 430 (1990).

[ACW90] S. Aggarwal, C. Courcoubetis, P. Wolper. "Adding Liveness Properties to Coupled FiniteState Machines", ACM TOPLAS, Vol. 12, No 2, April 1990.

[GK80] B. Gopinath, B. Kurshan. "The Selection/Resolution Model for Coordinating Concurrent Processes", AT\&T Bell Laboratories Technical Report.

[KK86] J. Katzenelson, B. Kurshan, "S/R: A Language for Specifying Protocols and other Coordinating Processes", Proc. 5th Ann. Int'l Phoenix Conf. Comput. Commun., IEEE, 1986.

[BGFRS90] A. Bouajjani, J.-C. Fernandez, S. Graf, C. Rodriguez, J. Sifakis. Safety for Branching Semantics, ICALP 91, Madrid, LNCS Vol. 510, 1991.

[Ma91] F. Maraninchi. Argos: a graphical synchronous language for the description of reactive systems, Report Spectre C-29, Grenoble, March 91, submitted to SCP

[Mi80] R. Milner. A Calculus for Communicating Systems, LNCS 92, 1980

[Mi83] R. Milner. Calculi for Synchrony and Asynchrony, Theoret. Comp. Sci. 25, 1983.

[Mi84] R. Milner. A Complete Inference System for a Class of Regular Behaviours, Journal of Comp. and Syst. Sci. Vol. 28, 1984

[Mi89] R. Milner. Communication and Concurrency, Prentice Hall, 1989 